\title{
Non-orthogonal Multiple Access in Large-Scale
}

\section{Underlay Cognitive Radio Networks}

\author{
Yuanwei Liu, Zhiguo Ding, Maged Elkashlan, and Jinhong Yuan
}

\begin{abstract}
In this paper, non-orthogonal multiple access (NOMA) is applied to large-scale underlay cognitive radio (CR) networks with randomly deployed users. In order to characterize the performance of the considered network, new closed-form expressions of the outage probability are derived using stochastic-geometry. More importantly, by carrying out the diversity analysis, new insights are obtained under the two scenarios with different power constraints: 1) fixed transmit power of the primary transmitters (PTs), and 2) transmit power of the PTs being proportional to that of the secondary base station. For the first scenario, a diversity order of $m$ is experienced at the $m$-th ordered NOMA user. For the second scenario, there is an asymptotic error floor for the outage probability. Simulation results are provided to verify the accuracy of the derived results. A pivotal conclusion is reached that by carefully designing target data rates and power allocation coefficients of users, NOMA can outperform conventional orthogonal multiple access in underlay CR networks.
\end{abstract}

\section{Index Terms}

Cognitive radio, large-scale network, non-orthogonal multiple access, stochastic geometry

\section{INTRODUCTION}

Spectrum efficiency is of significant importance and becomes one of the main design targets for future fifth generation networks. Non-orthogonal multiple access (NOMA) has received considerable attention because of its potential to achieve superior spectral efficiency [1]. Particularly, different from conventional multiple access (MA) techniques, NOMA uses the power domain to serve multiple users at different power levels in order to use spectrum more efficiently. A downlink NOMA and an uplink NOMA are considered in [2] and [3], respectively. The application of multiple-input multiple-output (MIMO) techniques to NOMA has been considered in [4] by using zero-forcing detection matrices. The authors in [5] investigated an ergodic capacity maximization problem for MIMO NOMA systems.

Y. Liu and M. Elkashlan are with Queen Mary University of London, London, UK (email: \{yuanwei.liu, maged.elkashlan\}@qmul.ac.uk).

Z. Ding is with Lancaster University, Lancaster, UK (e-mail: z.ding@lancaster.ac.uk).

J. Yuan is with the University of New South Wales, Sydney, Australia (e-mail: j.yuan@unsw.edu.au). 
Another approach to improve spectrum efficiency is the paradigm of underlay cognitive radio (CR) networks, which was proposed in [6] and has rekindled increasing interest in using spectrum more efficiently. The key idea of underlay CR networks is that each secondary user (SU) is allowed to access the spectrum of the primary users (PUs) as long as the SU meets a certain interference threshold in the primary network (PN). In [7], an underlay CR network taking into account the spatial distribution of the SU relays and PUs was considered and its performance was evaluated by using stochastic geometry tools. In [8], a new CR inspired NOMA scheme has been proposed and the impact of user pairing has been examined, by focusing on a simple scenario with only one primary transmitter (PT).

By introducing the aforementioned two concepts, it is natural to consider the application of NOMA in underlay CR networks using additional power control at the secondary base station (BS) to improve the spectral efficiency. Stochastic geometry is used to model a large-scale CR network with a large number of randomly deployed PTs and primary receivers (PRs). We consider a practical system design as follows: 1) All the SUs, PTs, and PRs are randomly deployed based on the considered stochastic geometry model; 2) Each SU suffers interference from other NOMA SUs as well as the PTs; and 3) The secondary BS must satisfy a predefined power constraint threshold to avoid interference at the PRs. New closed-form expressions of the outage probability of the NOMA users are derived to evaluate the performance of the considered CR NOMA network. Moreover, considering two different power constraints at the PTs, diversity order ${ }^{1}$ analysis is carried out with providing important insights: 1) When the transmit power of the PTs is fixed, the $m$-th user among all ordered NOMA user experiences a diversity order of $m$; and 2) When the the transmit power of the PTs is proportional to that of the secondary BS, an asymptotic error floor exists for the outage probability.

\section{NETWORK MODEL}

We consider a large-scale underlay spectrum sharing scenario consisting of the PN and the secondary network (SN). In the SN, we consider that a secondary BS is located at the origin of a disc, denoted by $\mathcal{D}$ with radius $R_{D}$ as its coverage. The $M$ randomly deployed secondary users are uniformly distributed

${ }^{1}$ Diversity order is defined as the slope for the outage provability curve decreasing with the signal-to-noise-ratio (SNR). It measures the number of independent fading paths over which the data is received. In NOMA networks, since users' channels are ordered and SIC is applied at each receiver, it is of importance to investigate the diversity order. 
within the disc which is the user zone for NOMA. The secondary BS communicates with all SUs within the disc by applying the NOMA transmission protocol. It is worthy pointing out that the power of the secondary transmitter is constrained in order to limit the interference at the PRs. In the PN, we consider a random number of PTs and PRs distributed in an infinite two dimensional plane. The spatial topology of all the PTs and PRs are modeled using homogeneous poisson point processes (PPPs), denoted by $\Phi_{b}$ and $\Phi_{\ell}$ with density $\lambda_{b}$ and $\lambda_{\ell}$, respectively. All channels are assumed to be quasi-static Rayleigh fading where the channel coefficients are constant for each transmission block but vary independently between different blocks.

According to underlay $\mathrm{CR}$, the transmit power $P_{t}$ at the secondary $\mathrm{BS}$ is constrained as follows:

$$
P_{t}=\min \left\{\frac{I_{p}}{\max _{\ell \in \Phi_{\ell}}\left|g_{\ell}\right|^{2}}, P_{s}\right\},
$$

where $I_{p}$ is the maximum permissible interference power at the PRs, $P_{s}$ is maximum transmission power at the secondary BS, $\left|g_{\ell}\right|^{2}=\left|\hat{g}_{\ell}\right|^{2} L\left(d_{\ell}\right)$ is the overall channel gain from the secondary BS to PRs $\ell$. Here, $\hat{g}_{\ell}$ is small-scale fading with $\hat{g}_{\ell} \sim \mathcal{C N}(0,1), L\left(d_{\ell}\right)=\frac{1}{1+d_{\ell}^{\alpha}}$ is large-scale path loss, $d_{\ell}$ is the distance between the secondary BS and the PRs, and $\alpha$ is the path loss exponent. A bounded path loss model is used to ensure the path loss is always larger than one even for small distances $[2,9]$.

According to NOMA, the BS sends a combination of messages to all NOMA users, and the observation at the $m$-th secondary user is given by

$$
y_{m}=h_{m} \sum_{n=1}^{M} \sqrt{a_{n} P_{t}} x_{n}+n_{m},
$$

where $n_{m}$ is the additive white Gaussian noise (AWGN) at the $m$-th user with variance $\sigma^{2}, a_{n}$ is the power allocation coefficient for the $n$-th SU with $\sum_{n=1}^{M} a_{n}=1, x_{n}$ is the information for the $n$-th user, and $h_{m}$ is the channel coefficient between the $m$-th user and the secondary BS.

For the SUs, they also observe the interferences of the randomly deployed PTs in the PN. Usually, when the PTs are close to the secondary NOMA users, they will cause significant interference. To overcome this issue, we introduce an interference guard zone $D_{0}$ to each secondary NOMA user with radius of $d_{0}$, which means that there is no interference from PTs allowed inside this zone [10]. We assume $d_{0} \geq 1$ in 
this paper. The interference links from the PTs to the SUs are dominated by the path loss and is given by

$$
I_{B}=\sum_{b \in \Phi_{b}} L\left(d_{b}\right)
$$

where $L\left(d_{b}\right)=1 /\left(1+d_{b}^{\alpha}\right)$ is the large-scale path loss and $d_{b}$ is the distance from the PTs to the SUs.

Without loss of generality, all the channels of SUs are assumed to follow the order as $\left|h_{1}\right|^{2} \leq\left|h_{2}\right|^{2} \leq$ $\cdots \leq\left|h_{M}\right|^{2}$. The power allocation coefficients are assumed to follow the order as $a_{1} \geq a_{2} \geq \cdots \geq a_{M}$. According to the NOMA principle, successive interference cancelation (SIC) is carried out at the receivers [11]. It is assumed that $1 \leq j \leq m<i$. In this case, the $m$-th user can decode the message of the $j$-th user and treats the message for the $i$-th user as interference. Specifically, the $m$-th user first decodes the messages of all the $(m-1)$ users, and then successively subtracts these messages to obtain its own information. Therefore, the received signal-to-interference-plus-noise ratio (SINR) for the $m$-th user to decode the information of the $j$-th user is given by

$$
\gamma_{m, j}=\frac{\left|h_{m}\right|^{2} \gamma_{t} a_{j}}{\left|h_{m}\right|^{2} \gamma_{t} \sum_{i=j+1}^{M} a_{i}+\rho_{b} I_{B}+1}
$$

where $\gamma_{t}=\min \left\{\frac{\rho_{p}}{\max _{\ell \in \Phi_{\ell}}\left|g_{\ell}\right|^{2}}, \rho_{s}\right\}, \rho_{p}=\frac{I_{p}}{\sigma^{2}}, \rho_{s}=\frac{P_{s}}{\sigma^{2}}, \rho_{b}=\frac{P_{B}}{\sigma^{2}}$, and $P_{B}$ is the transmit power of the PTs, $\left|h_{m}\right|^{2}$ is the overall ordered channel gain from the secondary BS to the $m$-th SU. For the case $m=j$, it indicates the $m$-th user decodes the message of itself. Note that the SINR for the $M$-th SU is $\gamma_{M, M}=\frac{\left|h_{M}\right|^{2} \gamma_{t} a_{M}}{\rho_{b} I_{B}+1}$.

\section{OUtAge Probability}

In this section, we provide exact analysis of the considered networks in terms of outage probability. In NOMA, an outage occurs if the $m$-th user can not detect any of the $j$-th user's message, where $j \leq m$ due to the SIC. Denote $X_{m}=\frac{\left|h_{m}\right|^{2} \gamma_{t}}{\rho_{b} I_{B}+1}$. Based on (4), the cumulative distribution function (CDF) of $X_{m}$ is given by

$$
F_{X_{m}}(\varepsilon)=\operatorname{Pr}\left\{\frac{\left|h_{m}\right|^{2} \gamma_{t}}{\rho_{b} I_{B}+1}<\varepsilon\right\}
$$

We denote $\varepsilon_{j}=\tau_{j} /\left(a_{j}-\tau_{j} \sum_{i=j+1}^{M} a_{i}\right)$ for $j<M, \tau_{j}=2^{R_{j}}-1, R_{j}$ is the target data rate for the $j$-th user, $\varepsilon_{M}=\tau_{M} / a_{M}$, and $\varepsilon_{m}^{\max }=\max \left\{\varepsilon_{1}, \varepsilon_{2}, \ldots, \varepsilon_{m}\right\}$. The outage probability at the $m$-th user can be expressed as follows:

$$
P_{m}=\operatorname{Pr}\left\{X_{m}<\varepsilon_{m}^{\max }\right\}=F_{X_{m}}\left(\varepsilon_{m}^{\max }\right)
$$


where the condition $a_{j}-\tau_{j} \sum_{i=j+1}^{M} a_{i}>0$ should be satisfied due to applying NOMA, otherwise the outage probability will always be one [2].

We need calculate the CDF of $X_{m}$ conditioned on $I_{B}$ and $\gamma_{t}$. Rewrite (5) as follows:

$$
F_{X_{m} \mid I_{B}, \gamma_{t}}(\varepsilon)=F_{\left|h_{m}\right|^{2}}\left(\frac{\left(\rho_{b} I_{B}+1\right) \varepsilon}{\gamma_{t}}\right)
$$

where $F_{\left|h_{m}\right|^{2}}$ is the CDF of $h_{m}$. Based on order statistics [12] and applying binomial expansion, the CDF of the ordered channels has a relationship with the unordered channels as follows:

$$
F_{\left|h_{m}\right|^{2}}(y)=\psi_{m} \sum_{p=0}^{M-m}\left(\begin{array}{c}
M-m \\
p
\end{array}\right) \frac{(-1)^{p}}{m+p}\left(F_{|\tilde{h}|^{2}}(y)\right)^{m+p},
$$

where $y=\frac{\left(\rho_{b} I_{B}+1\right) \varepsilon}{\gamma_{t}}, \psi_{m}=\frac{M !}{(M-m) !(m-1) !}$, and $|\tilde{h}|^{2}=|\hat{h}|^{2} L(d)$ is the unordered channel gain of an arbitrary SU. Here, $\hat{h}$ is the small-scale fading coefficient with $\hat{h} \sim \mathcal{C N}(0,1), L(d)=\frac{1}{1+d^{\alpha}}$ is the largescale path loss, and $d$ is a random variable representing the distance from the secondary BS to an arbitrary SU.

Then using the assumption of homogenous PPP and applying the polar coordinates, we express $F_{|\tilde{h}|^{2}}(y)$ as follows:

$$
F_{|\tilde{h}|^{2}}(y)=\frac{2}{R_{D}^{2}} \int_{0}^{R_{D}}\left(1-e^{-\left(1+r^{\alpha}\right) y}\right) r d r .
$$

Note that it is challenging to obtain an insightful expression for the unordered CDF. As such, we apply the Gaussian-Chebyshev quadrature [13] to find an approximation for (9) as

$$
F_{|\tilde{h}|^{2}}(y) \approx \sum_{n=0}^{N} b_{n} e^{-c_{n} y}
$$

where $N$ is a complexity-accuracy tradeoff parameter, $b_{n}=-\omega_{N} \sqrt{1-\phi_{n}^{2}}\left(\phi_{n}+1\right), b_{0}=-\sum_{n=1}^{N} b_{n}, c_{n}=$ $1+\left(\frac{R_{D}}{2}\left(\phi_{n}+1\right)\right)^{\alpha}, \omega_{N}=\frac{\pi}{N}$, and $\phi_{n}=\cos \left(\frac{2 n-1}{2 N} \pi\right)$.

Substituting (10) into (8) and applying the multinomial theorem, we obtain

$$
F_{\left|h_{m}\right|^{2}}(y)=\psi_{m} \sum_{p=0}^{M-m}\left(\begin{array}{c}
M-m \\
p
\end{array}\right) \frac{(-1)^{p}}{m+p} \sum_{q_{0}+\cdots+q_{N}=m+p}\left(\begin{array}{c}
m+p \\
q_{0}+\cdots+q_{N}
\end{array}\right)\left(\prod_{n=0}^{N} b_{n}^{q_{n}}\right) e^{-\sum_{n=0}^{N} q_{n} c_{n} y} .
$$


where $\left(\begin{array}{c}m+p \\ q_{0}+\cdots+q_{N}\end{array}\right)=\frac{(m+p) !}{q_{0} ! \cdots q_{N} !}$. Based on (11), the CDF of $X_{m}$ can be expressed as follows:

$$
\begin{aligned}
F_{X_{m}}\left(\varepsilon_{j}\right)= & \int_{0}^{\infty} \int_{0}^{\infty} F_{\left|h_{m}\right|^{2}}\left(\frac{\left(\rho_{b} x+1\right) \varepsilon_{j}}{z}\right) f_{I_{B}}(x) f_{\gamma_{t}}(z) d x d z \\
= & \psi_{m} \sum_{p=0}^{M-m}\left(\begin{array}{c}
M-m \\
p
\end{array}\right) \frac{(-1)^{p}}{m+p} \sum_{q_{0}+\cdots+q_{N}=m+p}\left(\begin{array}{c}
m+p \\
q_{0}+\cdots+q_{N}
\end{array}\right)\left(\prod_{n=0}^{N} b_{n}^{q_{n}}\right) \\
& \times \underbrace{\int_{0}^{\infty} e^{-\frac{\varepsilon_{j}}{z} \sum_{n=0}^{N} q_{n} c_{n}} \underbrace{\int_{0}^{\infty} e^{-\frac{x \rho_{b} \varepsilon_{j}}{z} \sum_{n=0}^{N} q_{n} c_{n}} f_{I_{B}}(x) d x}_{Q_{2}} f_{\gamma_{t}}(z) d z}_{Q_{1}}
\end{aligned}
$$

where $f_{\gamma_{t}}$ is the PDF of $\gamma_{t}$. We express $Q_{2}$ in (12) as follows:

$$
Q_{2}=\int_{0}^{\infty} e^{-x \frac{\rho_{b} \varepsilon_{j}}{z} \sum_{n=0}^{N} q_{n} c_{n}} f_{I_{B}}(x) d x=E_{\Phi_{b}}\left\{e^{-\frac{x \rho_{b} \varepsilon_{j}}{z} \sum_{n=0}^{N} q_{n} c_{n}}\right\}=L_{I_{B}}\left(\frac{x \rho_{b} \varepsilon_{j}}{z} \sum_{n=0}^{N} q_{n} c_{n}\right) .
$$

In this case, the Laplace transformation of the interferences from the PT can be expressed as [10]

$$
\begin{aligned}
L_{I_{B}}(s) & =\exp \left(-\lambda_{b} \pi\left[\left(e^{-s d_{0}^{-\alpha}}-1\right) d_{0}^{2}+s^{\delta} \gamma\left(1-\delta, s d_{0}^{-\alpha}\right)\right]\right) \\
& =\exp \left(-\lambda_{b} \pi[\left(e^{-s d_{0}^{-\alpha}}-1\right) d_{0}^{2}+s^{\delta} \underbrace{\int_{0}^{s d_{0}^{-\alpha}} t^{-\delta} e^{-t} d t}_{\Theta}]\right),
\end{aligned}
$$

where $\delta=\frac{2}{\alpha}$ and $\gamma(\cdot)$ is the lower incomplete Gamma function.

To obtain an insightful expression, we use Gaussian-Chebyshev quadrature to approximate the lower incomplete Gamma function in (14), $\Theta$ can be expressed as follows:

$$
\Theta \approx s^{1-\delta} \sum_{l=1}^{L} \beta_{l} e^{-t_{l} s d_{0}^{-\alpha}}
$$

where $L$ is a complexity-accuracy tradeoff parameter, $\beta_{l}=\frac{1}{2} d_{0}^{2-\alpha} \omega_{L} \sqrt{1-\theta_{l}^{2}} t_{l}^{-\delta}, t_{l}=\frac{1}{2}\left(\theta_{l}+1\right), \omega_{L}=\frac{\pi}{L}$, and $\theta_{l}=\cos \left(\frac{2 l-1}{2 L} \pi\right)$. Substituting (15) into (14), we approximate the Laplace transformation as follows:

$$
L_{I_{B}}(s) \approx e^{-\lambda_{b} \pi\left(\left(e^{-s d_{0}^{-\alpha}}-1\right) d_{0}^{2}+s \sum_{l=1}^{L} \beta_{l} e^{-t_{l} s d_{0}^{-\alpha}}\right)} .
$$

Substituting (16) into (12), $Q_{2}$ is given by

$$
Q_{2}=e^{-\lambda_{b} \pi\left(\left(e^{-\frac{\rho_{b} \varepsilon_{j} d_{0}^{-\alpha}}{z} \sum_{n=0}^{N} q_{n} c_{n}}-1\right) d_{0}^{2}+\frac{\rho_{b} \varepsilon_{j}}{z} \sum_{n=0}^{N} q_{n} c_{n} \sum_{l=1}^{L} \beta_{l} e^{-\frac{t_{l} \rho_{b} \varepsilon_{j}}{z d_{0}^{\alpha}} \sum_{n=0}^{N} q_{n} c_{n}}\right)} .
$$

The following theorem provides the PDF of $\gamma_{t}$. 
Theorem 1: Consider the use of the composite channel model with Rayleigh fading and path loss, the PDF of the effective power of the secondary BS is given by

$$
f_{\gamma_{t}}(x)=e^{-a_{\ell} \rho_{s}^{\delta} e^{-\frac{\rho_{p}}{x}}} \operatorname{Dirac}\left(x-\rho_{s}\right)+\left(\frac{\rho_{p}}{x}+\delta\right) a_{\ell} x^{\delta-1} e^{-a_{\ell} x^{\delta} e^{-\frac{\rho_{p}}{x}}-\frac{\rho_{p}}{x}} U\left(\rho_{s}-x\right),
$$

where $a_{\ell}=\frac{\delta \pi \lambda_{\ell} \Gamma(\delta)}{\rho_{p}^{\delta}}, U(\cdot)$ is the unit step function, and $\operatorname{Dirac}(\cdot)$ is the impulse function.

Proof: See Appendix A.

Substituting (18) and (17) into (12), we express $Q_{1}$ as follows:

$$
\begin{aligned}
& Q_{1}=e^{-a_{\ell} \rho_{s}^{\delta} e^{-\frac{\rho_{p}}{\rho_{s}}}-\frac{\varepsilon_{j} \sum_{n=0}^{N} q_{n} c_{n}}{\rho_{s}}-\lambda_{b} \pi}\left(\left(e^{-\frac{\rho_{b} \varepsilon_{j}}{\rho_{s} d_{0}^{\alpha}} \sum_{n=0}^{N} q_{n} c_{n}}-1\right) d_{0}^{2}+\frac{\rho_{b} \varepsilon_{j}}{\rho_{s}} \sum_{n=0}^{N} q_{n} c_{n} \sum_{l=1}^{L} \beta_{l} e^{-\frac{t_{l} \rho_{b} \varepsilon_{j}}{\rho_{s} d_{0}^{\alpha}} \sum_{n=0}^{N} q_{n} c_{n}}\right) \\
& +\underbrace{\int_{0}^{\rho_{s}} a_{\ell}\left(\frac{\rho_{p}}{z}+\delta\right) z^{\delta-1} e^{-a_{\ell} z^{\delta} e^{-\frac{\rho_{p}}{z}}-\frac{\rho_{p}+\varepsilon_{j}}{\sum_{n=0}^{N} q_{n} c_{n}}} Q_{2} d z}_{\Psi}
\end{aligned}
$$

We notice that it is very challenging to solve the integral $\Psi$ in (19), therefore, we apply the GaussianChebyshev quadrature to approximate the integral as follows:

$$
\Psi \approx \sum_{k=1}^{K} \eta_{k} e^{-\frac{\rho_{p}+\varepsilon_{j}}{\sum_{n=0}^{N} q_{n} c_{n}}}-\lambda_{b} \pi\left(\left(e^{-\frac{\rho_{b} \varepsilon_{j}}{\rho_{s} s_{k} d_{0}^{\alpha}} \sum_{n=0}^{N} q_{n} c_{n}}-1\right) d_{0}^{2}+\frac{\rho_{b} \varepsilon_{j}}{\rho_{s} s_{k}} \sum_{n=0}^{N} q_{n} c_{n} \sum_{l=1}^{L} \beta_{l} e^{-\frac{t_{l} \rho_{\rho} \varepsilon_{j}}{\rho_{s} s_{k} d_{0}^{\alpha}} \sum_{n=0}^{N} q_{n} c_{n}}\right),
$$

where $K$ is a complexity-accuracy tradeoff parameter, $\omega_{K}=\frac{\pi}{K}, \varphi_{k}=\cos \left(\frac{2 k-1}{2 K} \pi\right), s_{k}=\frac{1}{2}\left(\varphi_{k}+1\right)$, and $\eta_{k}=\frac{\omega_{K}}{2} \sqrt{1-\varphi_{k}^{2}}\left(\frac{\rho_{p}}{\rho_{s} s_{k}}+\delta\right) a_{\ell} \rho_{s}^{\delta} s_{k}^{\delta-1} e^{-a_{\ell} \rho_{s}^{\delta} s_{k}^{\delta} e^{-\frac{\rho p}{\rho_{s} s_{k}}}}$.

Substituting (19) and (20) into (12) and applying $\varepsilon_{\max } \rightarrow \varepsilon_{j}$, based on (6), we obtain the closed-form expression of the outage probability at the $m$-th user as follows:

$$
\begin{aligned}
& P_{m}=\psi_{m} \sum_{p=0}^{M-m}\left(\begin{array}{c}
M-m \\
p
\end{array}\right) \frac{(-1)^{p}}{m+p} \sum_{q_{0}+\cdots+q_{N}=m+p}\left(\begin{array}{c}
m+p \\
q_{0}+\cdots+q_{N}
\end{array}\right)\left(\prod_{n=0}^{N} b_{n}^{q_{n}}\right)
\end{aligned}
$$

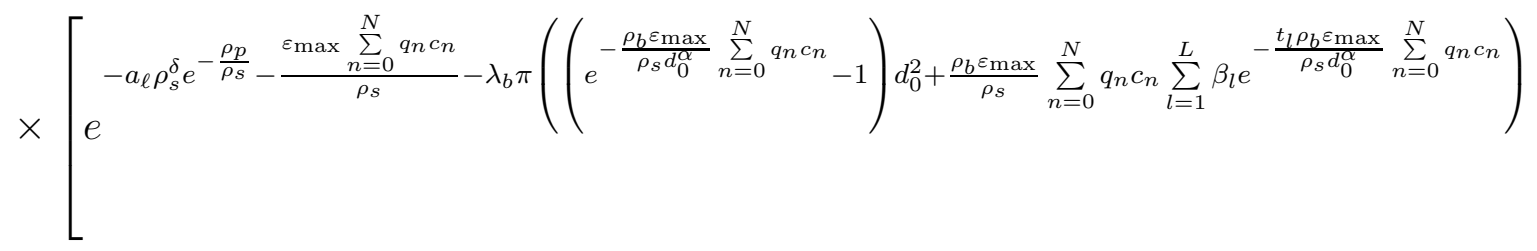

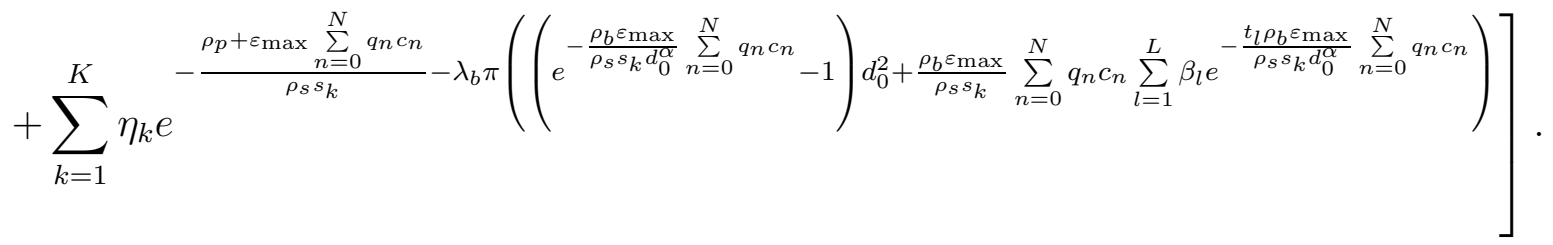




\section{Diversity ANALYSIS}

Based on the analytical results for the outage probability in (21), we aim to provide asymptotic diversity analysis for the ordered NOMA users. The diversity order of the user's outage probability is defined as

$$
d=-\lim _{\rho_{s} \rightarrow \infty} \frac{\log P_{m}\left(\rho_{s}\right)}{\log \rho_{s}} .
$$

\section{A. Fixed Transmit Power at Primary Transmitters}

In this case, we examine the diversity with the fixed transmit SNR at the PTs $\left(\rho_{b}\right)$, while the transmit SNR of secondary BS $\left(\rho_{s}\right)$ and the maximum permissible interference constraint at the PRs $\left(\rho_{p}\right)$ go to the infinity. Particularly, we assume $\rho_{p}$ is proportional to $\rho_{s}$, i.e. $\rho_{p}=\kappa \rho_{s}$, where $\kappa$ is a positive scaling factor. This assumption applies to the scenario where the PRs can tolerate a large amount of interference from the secondary BS and the target data rate is relatively small in the PN. Denote $\gamma_{t^{*}}=\frac{\gamma_{t}}{\rho_{s}}=\min \left\{\frac{\kappa}{\max _{\ell \in \Phi_{\ell}}\left|g_{\ell}\right|^{2}}, 1\right\}$, similar to (8), the ordered $\mathrm{CDF}$ has the relationship with unordered $\mathrm{CDF}$ as

$$
F_{X_{m} \mid I_{B}, \gamma_{t^{*}}}^{\infty}\left(y^{*}\right)=\psi_{m} \sum_{p=0}^{M-m}\left(\begin{array}{c}
M-m \\
p
\end{array}\right) \frac{(-1)^{p}}{m+p}\left(F_{|\tilde{h}|^{2}}^{\infty}\left(y^{*}\right)\right)^{m+p}
$$

where $y^{*}=\frac{\left(\rho_{b} I_{B}+1\right) \varepsilon_{j}}{\rho_{s} \gamma_{t^{*}}}$. When $\rho_{s} \rightarrow \infty$, we observe that $y^{*} \rightarrow 0$. In order to investigate an insightful expression to obtain the diversity order, we use Gaussian-Chebyshev quadrature and $1-e^{-y^{*}} \approx y^{*}$ to approximate (9) as

$$
F_{|\tilde{h}|^{2}}^{\infty}\left(y^{*}\right) \approx \sum_{n=1}^{N} \chi_{n} y^{*}
$$

where $\chi_{n}=\omega_{N} \sqrt{1-\phi_{n}^{2}}\left(\phi_{n}+1\right) c_{n}$. Substituting (24) into (23), since $y^{*} \rightarrow 0$, we obtain

$$
F_{X_{m} \mid I_{B}, \gamma_{t^{*}}}^{\infty}\left(\varepsilon_{j}\right)=\xi\left(\frac{\left(\rho_{b} I_{B}+1\right) \varepsilon_{j}}{\rho_{s} \gamma_{t^{*}}}\right)^{m}+o\left(\left(\frac{\left(\rho_{b} I_{B}+1\right) \varepsilon_{j}}{\rho_{s} \gamma_{t^{*}}}\right)^{m}\right)
$$

where $\xi=\frac{\psi_{m}\left(\sum_{n=1}^{N} \chi_{n}\right)^{m}}{m}$. Based on (6), (11), and (25), the asymptotic outage probability is given by

$$
P_{m_{F}}^{\infty} \approx \frac{1}{\rho_{s}^{m}} \underbrace{\int_{0}^{\infty} \int_{0}^{\infty} \xi\left(\frac{\left(\rho_{b} x+1\right) \varepsilon_{\max }}{z}\right)^{m} f_{I_{B}}(x) f_{\gamma_{t^{*}}}(z) d x d z}_{C}
$$

where $f_{\gamma_{t^{*}}}$ the PDF of $\gamma_{t^{*}}$. Since $C$ is a constant independent of $\rho_{s}$, (26) can be expressed as follows:

$$
P_{m_{F}}^{\infty}=\frac{1}{\rho_{s}^{m}} C+o\left(\rho_{s}{ }^{-m}\right)
$$


Substituting (27) into (22), we obtain the diversity order of this case is $m$. This can be explained as follows. Note that SIC is applied at the ordered SUs. For the first user with the poorest channel gain, no interference cancelation is operated at the receiver, therefore its diversity gain is one. While for the $m$-th user, since the interferences from all the other $(m-1)$ users are canceled, it obtains a diversity of $m$.

\section{B. Transmit Power of Primary Transmitters Proportional to that of Secondary Ones}

In this case, we examine the diversity with the transmit SNR at the PTs $\left(\rho_{b}\right)$ is proportional to the transmit SNR of secondary BS $\left(\rho_{s}\right)$. Particularly, we assume $\rho_{b}=\nu \rho_{s}$, where $\nu$ is a positive scaling factor. We still assume $\rho_{p}$ is proportional to $\rho_{s}$. Applying $\rho_{s} \rightarrow \infty, \rho_{p}=\kappa \rho_{s}$ and $\rho_{b}=\nu \rho_{s}$ to (21), we obtain the asymptotic outage probability of the $m$-th user in this case as follows:

$$
\begin{aligned}
& P_{m_{P}}^{\infty} \approx \psi_{m} \sum_{p=0}^{M-m}\left(\begin{array}{c}
M-m \\
p
\end{array}\right) \frac{(-1)^{p}}{m+p} \sum_{q_{0}+\cdots+q_{N}=m+p}\left(\begin{array}{c}
m+p \\
q_{0}+\cdots+q_{N}
\end{array}\right)\left(\prod_{n=0}^{N} b_{n}^{q_{n}}\right) \\
& \times\left[e^{-a_{\ell}^{\infty} e^{-\kappa}-\lambda_{b} \pi}\left(\left(e^{-\frac{\nu \varepsilon_{\max }}{d_{0}^{\alpha}} \sum_{n=0}^{N} q_{n} c_{n}}-1\right) d_{0}^{2}+\nu \varepsilon_{\max } \sum_{n=0}^{N} q_{n} c_{n} \sum_{l=1}^{L} \beta_{l} e^{-\frac{t_{l} \nu \varepsilon_{\max }}{d_{0}^{\alpha}} \sum_{n=0}^{N} q_{n} c_{n}}\right)\right.
\end{aligned}
$$

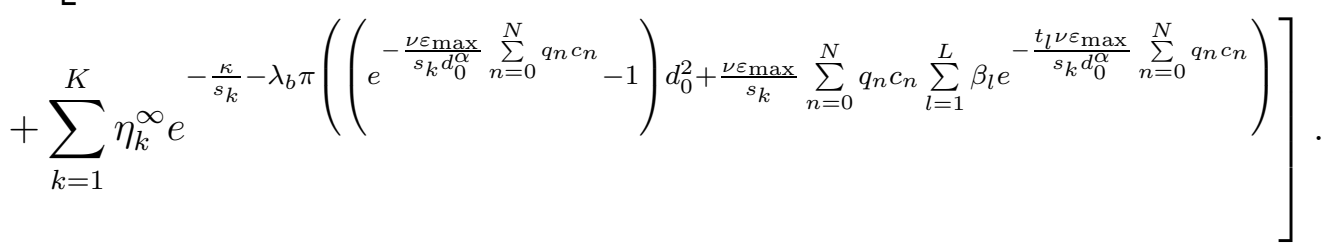

where $a_{\ell}^{\infty}=\frac{\delta \pi \lambda_{\ell} \Gamma(\delta)}{\kappa^{\delta}}$ and $\eta_{k}^{\infty}=\frac{\omega_{K}}{2} \sqrt{1-\varphi_{k}^{2}}\left(\frac{\kappa}{s_{k}}+\delta\right) a_{\ell}^{\infty} s_{k}^{\delta-1} e^{-a_{\ell}^{\infty} s_{k}^{\delta} e^{-\frac{\kappa}{s_{k}}}}$.

It is observed that $P_{m_{P}}^{\infty}$ is a constant independent of $\rho_{s}$. Substituting (28) into (22), we find that asymptotically there is an error floor for the outage probability of SUs.

\section{NumericAl RESUltS}

In this section, numerical results are presented to verify the accuracy of the analysis as well as to obtain more important insights for NOMA in large-scale CR networks. In the considered network, the radius of the guard zone is assumed to be $d_{0}=2 \mathrm{~m}$. The Gaussian-Chebyshev parameters are chosen with $N=5$, $K=10$, and $L=10$. Monte Carlo simulation results are marked as "•" to verify our derivation.

Fig. 1 plots the outage probability of the $m$-th user for the first scenario when $\rho_{b}$ is fixed and $\rho_{p}$ is proportional to $\rho_{s}$. In Fig. 1(a), the power allocation coefficients are $a_{1}=0.5, a_{2}=0.4$ and $a_{3}=0.1$. The target data rate for each user is assumed to be all the same as $R_{1}=R_{2}=R_{3}=0.1$ bit per channel use (BPCU). The dashed and solid curves are obtained from the analytical results derived in (21). Several 


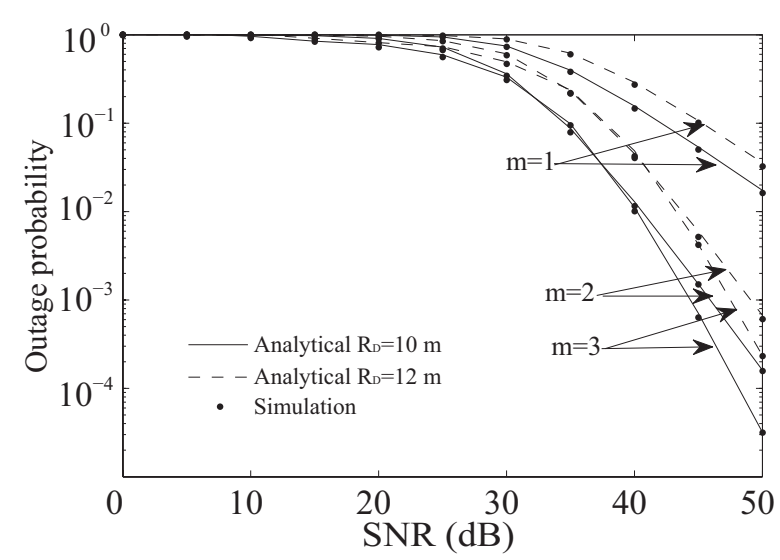

(a) For different user zone, with $\lambda_{b}=10^{-3}, \lambda_{\ell}=10^{-3}, \kappa=1$, $\alpha=4, \rho_{b}=20 \mathrm{~dB}$, and $M=3$.

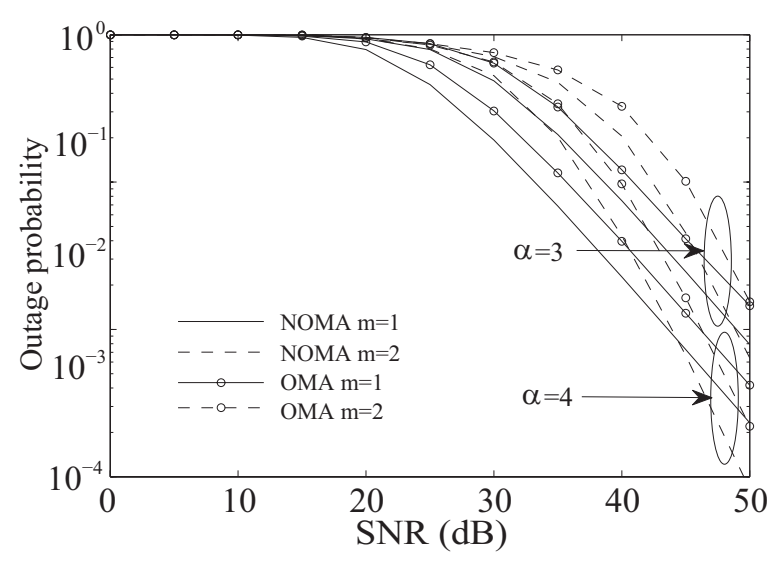

(b) For different $\alpha$, with $\lambda_{b}=10^{-3}, \lambda_{\ell}=10^{-3}, \kappa=1, R_{D}=$ $5 \mathrm{~m}, \rho_{b}=20 \mathrm{~dB}$, and $M=2$.

Fig. 1: Outage probability of the $m$-th user versus $\rho_{s}$ of the first scenario.

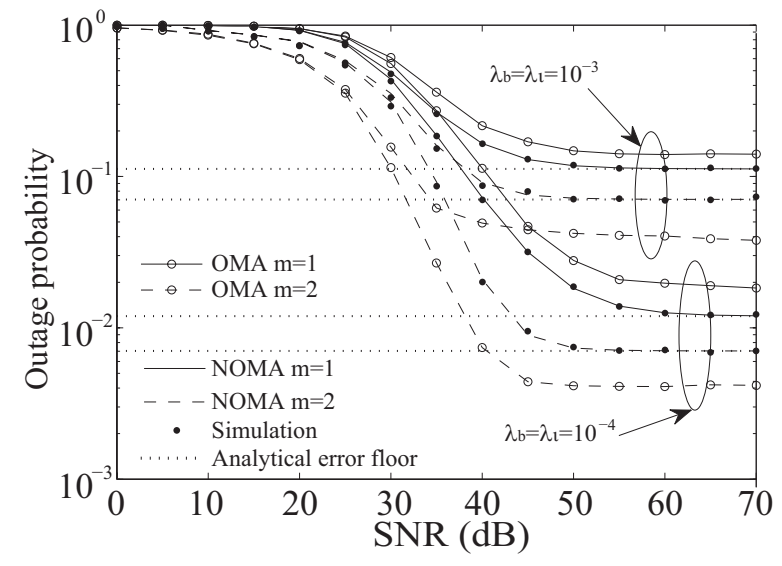

(a) For different density of PTs and PRs, with $\alpha=4, \kappa=1$, $\nu=1, R_{D}=10 \mathrm{~m}, \rho_{b}=\nu \rho_{s}$, and $M=2$.

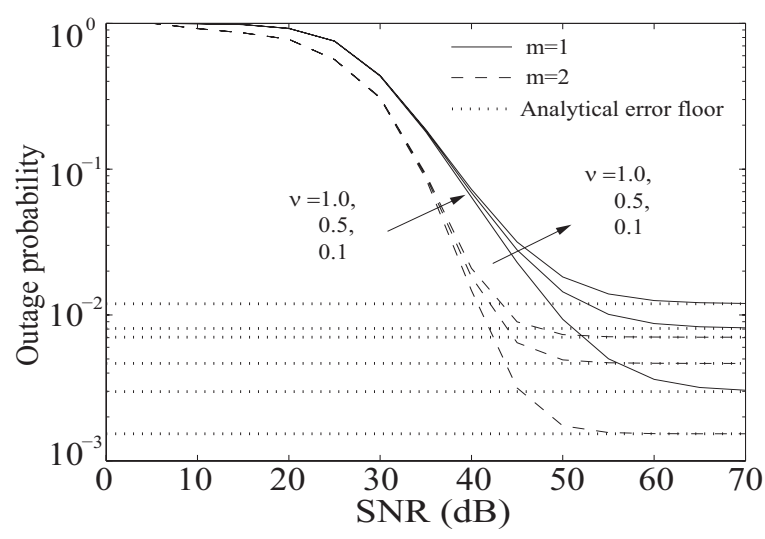

(b) For different $\nu$, with $\alpha=4, \lambda_{b}=10^{-4}, \lambda_{\ell}=10^{-4}, \kappa=0.5$, $R_{D}=10 \mathrm{~m}, \rho_{b}=\nu \rho_{s}$, and $M=2$.

Fig. 2: Outage probability of the $m$-th user versus $\rho_{s}$ of the second scenario.

observations can be drawn as follows: 1) Reducing the coverage of the secondary users zone $\mathcal{D}$ can achieve a lower outage probability because of a smaller path loss. 2) The ordered users with different channel conditions have different decreasing slope because of different diversity orders, which verifies the derivation of (26). In Fig. 1(b), the power allocation coefficients are $a_{1}=0.8$ and $a_{2}=0.2$. The target rate is $R_{1}=1$ and $R_{2}=3 \mathrm{BPCU}$. The performance of a conventional OMA is also shown in the figure as a benchmark for comparison. It can be observed that for different values of the path loss, NOMA can 
achieve a lower outage probability than the conventional OMA.

Fig. 2 plots the outage probability of the $m$-th user for the second scenario when both $\rho_{b}$ and $\rho_{p}$ are proportional to $\rho_{s}$. The power allocation coefficients are $a_{1}=0.8$ and $a_{2}=0.2$. The target rates are $R_{1}=R_{2}=0.1 \mathrm{BPCU}$. The dashed and solid curves are obtained from the analytical results derived in (21). One observation is that error floors exist in both Figs. 2(a) and 2(b), which verifies the asymptotic results in (28). Another observation is that user two $(m=2)$ outperforms user one $(m=1)$. The reason is that for user two, by applying SIC, the interference from user one is canceled. While for user one, the interference from user two still exists. In Fig. 2(a), it is shown that the error floor become smaller when $\lambda_{b}$ and $\lambda_{\ell}$ decrease, which is due to less interference from PTs and the relaxed interference power constraint at the PRs. It is also worth noting that with these system parameters, NOMA outperforms OMA for user one while OMA outperforms NOMA for user two, which indicates the importance of selecting appropriate power allocation coefficients and target data rates for NOMA. In Fig. 2(b), it is observed that the error floors become smaller as $\nu$ decreases. This is due to the fact that smaller $\nu$ means a lower transmit power of PTs, which in turn reduces the interference at SUs.

\section{CONCLUSIONS}

In this paper, we have studied non-orthogonal multiple access (NOMA) in large-scale underlay cognitive radio networks with randomly deployed users. Stochastic geometry tools were used to evaluate the outage performance of the considered network. New closed-form expressions were derived for the outage probability. Diversity order of NOMA users has been analyzed in two situations based on the derived outage probability. An important future direction is to optimize the power allocation coefficients to further improve the performance gap between NOMA and conventional MA in CR networks.

\section{Appendix A: Proof of Theorem 1}

The CDF of $\gamma_{t}$ is given by

$$
\begin{aligned}
F_{\gamma_{t}}(x) & =\operatorname{Pr}\left\{\min \left\{\frac{\rho_{p}}{\max _{\ell \in \Phi_{\ell}}\left|g_{\ell}\right|^{2}}, \rho_{s}\right\} \leq x\right\} \\
& =\operatorname{Pr}\left\{\max _{\ell \in \Phi_{\ell}}\left|g_{\ell}\right|^{2} \geq \max \left\{\frac{\rho_{p}}{x}, \frac{\rho_{p}}{\rho_{s}}\right\}\right\}+\operatorname{Pr}\left\{\max _{\ell \in \Phi_{\ell}}\left|g_{\ell}\right|^{2} \leq \frac{\rho_{p}}{\rho_{s}}, \rho_{s} \leq x\right\} \\
& =1-U\left(\rho_{s}-x\right) \underbrace{\operatorname{Pr}\left\{\max _{\ell \in \Phi_{\ell}}\left|g_{\ell}\right|^{2} \geq \frac{\rho_{p}}{x}\right\}}_{\Omega} .
\end{aligned}
$$


Denote $\bar{\Omega}=1-\Omega$, we express $\bar{\Omega}$ as follows:

$$
\begin{aligned}
\bar{\Omega} & =\operatorname{Pr}\left\{\max _{\ell \in \Phi_{\ell}}\left|g_{\ell}\right|^{2} \leq \frac{\rho_{p}}{x}\right\}=E_{\Phi_{\ell}}\left\{\prod_{\ell \in \Phi_{\ell}} \operatorname{Pr}\left\{\left|\hat{g}_{\ell}\right|^{2} \leq \frac{\left(1+d_{\ell}^{\alpha}\right) \rho_{p}}{x}\right\}\right\} \\
& =E_{\Phi_{\ell}}\left\{\prod_{\ell \in \Phi_{\ell}} F_{\left|\hat{g}_{\ell}\right|^{2}}\left(\frac{\left(1+d_{\ell}^{\alpha}\right) \rho_{p}}{x}\right)\right\} .
\end{aligned}
$$

Applying the generating function, we rewrite (A.2) as follows:

$$
\bar{\Omega}=\exp \left[-\lambda_{\ell} \int_{R^{2}}\left(1-F_{\left|\hat{g}_{\ell}\right|^{2}}\left(\left(1+d_{\ell}^{\alpha}\right) \mu\right)\right) r d r\right]=\exp \left[-2 \pi \lambda_{\ell} e^{-\mu} \int_{0}^{\infty} r e^{-\mu r^{\alpha}} d r\right] .
$$

Applying [14, Eq. (3.326.2)], we obtain

$$
\Omega=1-\bar{\Omega}=1-e^{-\frac{e^{-\mu} \delta \pi \lambda_{\ell} \Gamma(\delta)}{\mu^{\delta}}},
$$

where $\Gamma(\cdot)$ is Gamma function. Substituting (A.4) into (A.1), and taking the derivative, we obtain the PDF of $\gamma_{t}$ in (18). The proof is completed.

\section{REFERENCES}

[1] Y. Saito, A. Benjebbour, Y. Kishiyama, and T. Nakamura, "System-level performance evaluation of downlink non-orthogonal multiple access (NOMA)," in Proc. IEEE Annual Symposium on Personal, Indoor and Mobile Radio Communications (PIMRC), Sept. 2013.

[2] Z. Ding, Z. Yang, P. Fan, and H. V. Poor, "On the performance of non-orthogonal multiple access in 5G systems with randomly deployed users," IEEE Signal Process. Lett., vol. 21, no. 12, pp. 1501-1505, 2014.

[3] M. Al-Imari, P. Xiao, M. A. Imran, and R. Tafazolli, "Uplink non-orthogonal multiple access for 5G wireless networks," in Proc. of the 11th International Symposium on Wireless Communications Systems (ISWCS), Barcelona, Spain, Aug 2014, pp. 781-785.

[4] Z. Ding, F. Adachi, and H. V. Poor, "The application of MIMO to non-orthogonal multiple access," IEEE Trans. Wireless Commun., 2016.

[5] Q. Sun, S. Han, C.-L. I, and Z. Pan, “On the ergodic capacity of MIMO NOMA systems,” IEEE Wireless Commun. Lett., 2015.

[6] A. Goldsmith, S. A. Jafar, I. Maric, and S. Srinivasa, "Breaking spectrum gridlock with cognitive radios: An information theoretic perspective," Proceedings of the IEEE, vol. 97, no. 5, pp. 894-914, 2009.

[7] Y. Dhungana and C. Tellambura, "Outage probability of underlay cognitive relay networks with spatially random nodes," in Global Commun. Conf. (GLOBECOM), Dec 2014, pp. 3597-3602.

[8] Z. Ding, P. Fan, and H. V. Poor, "Impact of user pairing on 5G non-orthogonal multiple access," IEEE Trans. Veh. Technol., 2016.

[9] M. Haenggi, Stochastic Geometry for Wireless Networks. Cambridge, U.K.: Cambridge Univ. Press, 2012.

[10] J. Venkataraman, M. Haenggi, and O. Collins, "Shot noise models for outage and throughput analyses in wireless ad hoc networks," in Military Commun. Conf. (MILCOM), 2006, pp. 1-7.

[11] T. M. Cover and J. A. Thomas, "Elements of information theory 2nd edition," 2006.

[12] H. A. David and N. Nagaraja, Order Statistics, 3rd ed. John Wiley, 2003.

[13] E. Hildebrand, "Introduction to numerical analysis," NewYork, NY, USA: Dover, 1987.

[14] I. S. Gradshteyn and I. M. Ryzhik, Table of Integrals, Series and Products, 6th ed. New York, NY, USA: Academic Press, 2000. 